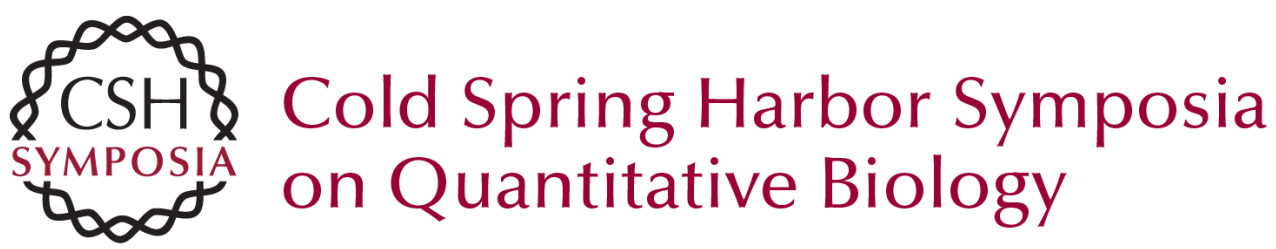

\title{
Protein-conducting Channels for the Translocation of Proteins into and across Membranes
}

S.M. Simon

Cold Spring Harb Symp Quant Biol 1995 60: 57-69

Access the most recent version at doi:10.1101/SQB.1995.060.01.009

References This article cites 55 articles, 28 of which can be accessed free at: http://symposium.cshlp.org/content/60/57.refs.html

Email alerting Receive free email alerts when new articles cite this article - sign up in service the box at the top right corner of the article or click here

To subscribe to Cold Spring Harbor Symposia on Quantitative Biology go to: http://symposium.cshlp.org/subscriptions 


\title{
Protein-conducting Channels for the Translocation of Proteins into and across Membranes
}

\author{
S.M. Simon \\ Laboratory of Cellular Biophysics, Rockefeller University, New York, New York 10021
}

The membranes surrounding cells and organelles are permeability barriers. Cell viability is dependent on maintaining membrane integrity and impermeability while simultaneously permitting regulated and selective transmembrane transport of substrates and effector molecules.

Most studies of membrane transport have focused on the movement of ions. Membranes are normally extremely impermeable to these molecules. There are two different systems for selective ion transport across membranes: channels and carriers. Channels allow ions to cross the membrane by free diffusion through aqueous pores. In contrast, carriers sequentially expose their transport-specific binding site to each side of the membrane. Carriers can vary from small molecules like valinomycin, which bind to the potassium ion at the membrane surface and can diffuse across the membrane, to the large $\mathrm{Na} / \mathrm{K}$ ATPase with many transmembrane domains. In contrast, channels are only transmembrane protejns, since they must maintain a clear aqueous pathway across the membrane for ion movement.

Our understanding of how channels work goes back to the work of Nernst and Planck over 100 years ago and has been substantially enhanced by the elegant electrophysiological studies of Hodgkin and Huxley and Neher and Sakman (for review, see Hille 1984). Considerably less is known of the mechanisms for membrane transport of larger molecules, such as amino acids, peptides, proteins, polynucleotides, or macromolecular complexes, across membranes.

It has been almost 30 years since it was demonstrated that secretory proteins translocate across the endoplasmic reticulum (ER) as they are being synthesized (Redman and Sabatini 1966). Five years later, the signal hypothesis was proposed as an intellectual framework for designing experiments to explore protein targeting to and translocation across membranes (Blobel and Sabatini 1971). Most of our current understanding of protein targeting to membranes was made possible by the development of in vitro systems for testing the predictions of this signal hypothesis (Blobel and Dobberstein 1975). The use of these systems has demonstrated that (1) the signal sequence, a linear stretch of amino acids in the protein, carries the information for targeting a protein for translocation into an organelle; (2) proteins must be unfolded in order to translocate; and (3) cytosolic fac- tors are necessary for both holding a nascent protein in an unfolded conformation and helping to target the nascent peptide to the organelle (for review, see Walter and Lingappa 1986).

The success of the signal hypothesis in describing protein targeting to organelles has created its own series of questions. Other papers in this volume address the issue of identifying the proteins that form the translocation site (see Blobel; Brown et al.; Panzner et al; all this volume). In this paper, I review some of the recent work performed in my laboratory to address two of these questions:

1. How does the protein traverse the membrane? Does it cross through the lipid or, like ions, does it move through an aqueous protein-conducting channel? What drives the protein across the membrane?

2. How are membrane proteins integrated into the bilayer?

Our results indicate that proteins, like ions, seem to cross the membrane through transmembrane aqueous pores. The signal sequence serves as the ligand to open these protein-conducting channels. As such, the protein-conducting channels are not unlike ligandgated ion channels. Upon opening, the nascent translocating protein occludes the lumen of the proteinconducting channels so that they are not freely permeable to ions. Similarly, ion channels are also occluded, blocked to the free flow of ions, when peptides enter their lumen (Zagotta et al. 1990).

What moves proteins across the membranes? Most, if not all, protein translocation can be explained by assuming that proteins fluctuate back and forth across the membrane in response to thermal energy (Simon et al. 1992). Any modifications of the protein in the ER lumen, such as glycosylation, binding of chaperones, or disulfide bond formation, provide an energetic barrier against backward fluctuation. Thus, the vectoriality of protein transport is the consequence of a combination of thermal fluctuations and an asymmetry of chemical potential (in the form of asymmetries in modifying enzymes, ionic gradients, etc.). Likewise, ion movement through ion channels is the consequence of thermal fluctuations of the ions combined with a chemical potential gradient. For the ions, the chemical potential gradient is primarily due to contributions from electrical and chemical (concentration) energy. Thus, although protein-conducting channels have only begun 
to be characterized, functionally they resemble the already well-understood ion-conducting channels.

There is one major potential difference between the two systems. Secretory proteins translocate across, perpendicular to the membrane surface. However, nascent membrane proteins must move in two dimensions: Each latent transmembrane segment must first move perpendicular to the membrane and then parallel to the surface membrane to integrate into the lipid bilayer. A description of how membrane proteins achieve their proper transmembrane topography, especially polytopic membrane proteins (those with many transmembrane segments) and oligomeric proteins (those with multiple subunits), are key challenges to our understanding of protein translocation.

\section{METHODS}

Materials. Rabbit reticulocyte lysate was obtained from Promega and protease inhibitors were obtained from Boehringer Mannheim. The transcription reactions were done with Ambion's SP6 MEGAscript kit. Restriction enzymes were ordered from either Boehringer Mannheim or New England Biolabs. We purchased cetyltrimethyl-ammonium bromide (CTABr) from Calbiochem and prepared a $2 \%$ stock. Lipids were obtained from Avanti Polar (Birmingham, Alabama). All other chemicals were obtained from Fisher or Sigma Chemical Company.

Bilayer formation. Planar phospholipid bilayers of bacterial phosphatidylethanolamine and bovine phosphatidylserine (Avanti Polar, Birmingham, Alabama), $20 \mathrm{mg} / \mathrm{ml}$ each in decane (Fluka), were made as described previously (Mueller et al. 1963; Simon and Blobel 1991) across a $200-\mu \mathrm{m}$ to $1-\mathrm{mm}$ hole in a KEL-F partition separating two chambers. The two chambers initially held $3 \mathrm{ml}$ of trans solution $(45 \mathrm{mM}$ potassium glutamate/5 mM HEPES· KOH [pH 7.5]/3 mM $\mathrm{MgCl}_{2}$ ). $A$ bilayer was used only if after initial formation it had a stable conductance of less than $10 \mathrm{pS}$ for at least 20 minutes. After bilayer formation, the cis chamber was made hyperosmotic by the addition of urea or sucrose to a final concentration of $300 \mathrm{mM}$ or $250 \mathrm{~mm}$, respectively.

Fusion of ER vesicles. Approximately $0.5 \mu \mathrm{l}$ of ER vesicles $\left(0.02 \quad \mathrm{~A}_{280} / \mathrm{ml}\right)$ was pressure-injected (Picospritzer II, General Valve Corporation, New Jersey) from a micropipet into the space adjacent to the cis face of the bilayer (Niles and Cohen 1987; Simon et al. 1989; Simon and Blobel 1991, 1992). The trans side refers to the opposite side of the membrane. In the presence of an osmotic gradient (Zimmerberg et al. 1980), ER vesicles fuse to the bilayer within seconds, which results in conductance increases that remain stable for hours (Simon et al. 1989). After fusion, the cis compartment was then perfused with $20 \mathrm{ml}$ of trans solution to eliminate the osmotic gradient and unfused ER vesicles. This was accomplished with a homemade device of two $20-\mathrm{ml}$ syringes connected plunger to plunger (C. Miller, pers. comm.). Stock solutions of 20 $\mathrm{mm}$ puromycin- $\mathrm{HCl}$ (Boehringer Mannheim and Calbiochem) were adjusted to $\mathrm{pH} 7.5$ with $\mathrm{KOH}$ and stored frozen in 20-ml aliquots. Puromycin was added to the chambers by hand with an Eppendorf pipet while both chambers were being stirred with two magnetic stir bars.

Electrophysiology. The voltage across the bilayer is applied via two calomel electrodes, which are connected to the cis and trans compartments via glass capillary tubes filled with $150 \mathrm{mM} \mathrm{KCl}$ in $1.5 \%$ agar. The voltage is imposed and the current is measured with a homemade voltage clamp using an operational amplifier (AD515 Analog Devices, Norwood, Massachusetts) with feedback resistors of $10^{8}(0.5 \%), 10^{9}$ $(0.5 \%)$, or $10^{10}(1 \%)$ ohms ( $\mathrm{K}$ and $\mathrm{M}$ Electronics Inc., Springfield, Massachusetts) or with an Axopatch $200 \mathrm{~A}$ voltage clamp (Axon Instruments, Foster City, California). The trans chamber is grounded and all voltages are recorded for the cis chamber relative to trans. All experiments were controlled via software that was written in the LabView programming environment (National Instruments). All data were digitized at 5 $\mathrm{kHz}$ (16-bit resolution) and filtered at $1 \mathrm{kHz}$ prior to digitizing with an 8-pole Bessel Filter (Frequency Devices, Norwood, Massachusetts).

Plasmids and mRNA transcription. The plasmid encoding the vesicular stomatitis virus glycoprotein was provided by Dr. Ari Helenius. Transcription reactions included the cap analog, $\mathrm{m}^{7} \mathrm{G}\left(5^{\prime}\right) \mathrm{ppp}\left(5^{\prime}\right) \mathrm{G}$ (Ambion) and were carried out with an SP6 MEGAscript kit (Ambion) following restriction enzyme cleavage of the plasmid to create runoff transcripts. The RNA was purified with phenol and chloroform extractions, precipitated, and aliquoted for storage.

Protein translations. In vitro translations were performed using Promega's Rabbit Reticulocyte Lysate (RRL) system with one-half volume of the suggested reticulocyte lysate supplemented with a translation/translocation buffer containing $120 \mathrm{mM} \mathrm{KOAc}$ $(\mathrm{pH} 7.5), 50 \mathrm{mM}$ HEPES $(\mathrm{pH} 7.5)$, and $2 \mathrm{~mm}$ $\mathrm{Mg}(\mathrm{OAc})_{2}$ to bring up the reaction volume. Each $25-\mu \mathrm{l}$ reaction contained $\left.30 \mu \mathrm{Ci}{ }^{35} \mathrm{~S}\right]$ methionine, 3.6 equivalents of canine pancreatic microsomal membranes, and 40 units of RNasin ribonuclease inhibitor and were carried out at $22^{\circ} \mathrm{C}$ for 50 minutes. Pancreatic microsomes were made as described previously (Walter and Blobel 1983). Cycloheximide (at a final concentration of $0.05 \mathrm{mg} / \mathrm{ml}$ ) or puromycin (at $2 \mathrm{mM}$ ) was added when specified and incubated at $22^{\circ} \mathrm{C}$ for 10 minutes.

Harvesting microsomal membranes. The translation products were layered on top of $100 \mu \mathrm{l}$ of $1.0 \mathrm{M}$ sucrose (with $20 \mathrm{mM} \mathrm{KHPO}_{4}$ [pH 7], $1 \mathrm{mM} \beta$-mercaptoethanol, and $2 \mathrm{~mm} \mathrm{Mg}[\mathrm{OAc}]_{2}$ ) and $10 \mu \mathrm{l}$ of $2 \mathrm{M}$ sucrose and centrifuged in a Beckman Optima TLX Ultracentrifuge at $186,000 \mathrm{~g}$ for 15 minutes at $4^{\circ} \mathrm{C}$ to harvest trans- 
lation products that were targeted to the ER vesicles. The top of the cushion was removed containing protein not associated with the membrane vesicles, precipitated with acetone, and examined by SDS-PAGE. The majority of the translation product was found in the 25 $\mu \mathrm{l}$ portion of the sucrose left behind, layered on top of the $2 \mathrm{M}$ sucrose cushion.

Precipitation of $t R N A$-bound peptides. CTABr is a detergent that efficiently precipitates tRNA-bound peptides. $250 \mu$ l of $2 \%$ CTABr was added to a $10-20-\mu \mathrm{l}$ translation reaction and vortexed, and $250 \mu \mathrm{l}$ of $0.5 \mathrm{M}$ sodium acetate ( $\mathrm{pH} 5.4$ ) containing $200 \mu \mathrm{g} / \mathrm{ml}$ yeast tRNA was added and revortexed. After incubation for 10 minutes at $30^{\circ} \mathrm{C}$, the samples were centrifuged for 10 minutes at $12,000 \mathrm{~g}$. The supernatant sample was precipitated with acetone, and the pellet sample was washed with acetone as well, and both were prepared for SDS-PAGE.

Gel electrophoresis and image analysis. Gels were prepared using 9-12\% acrylamide with $6 \mathrm{~m}$ urea. The gels were dried and exposed either to Kodak X-Omat AR-5 film at $-70^{\circ} \mathrm{C}$ or to a Molecular Dynamics phosphor screen. The scanned images were analyzed with Molecular Dynamics software.

\section{RESULTS}

\section{What Is the Environment for Protein Translocation?}

Do proteins translocate directly through the hydrocarbon core of the lipid bilayer, or through a transmembrane aqueous pathway? Specifically, our experiments were designed to test the hypothesis that translocation occurs through protein-conducting channels (Blobel and Dobberstein 1975). To address this question, we have used many of the same techniques used by electrophysiologists to study ion-conducting channels. We reasoned that protein-conducting channels should be considerably larger than ion-conducting channels and thus are likely to be detectable with the same electrophysiological tools and approaches.

To test for the presence of protein-conducting channels, we fused vesicles of pancreatic ER to a planar lipid bilayer that separates two aqueous chambers. A number of large channels were observed. These channels were large, as characterized either by the magnitude of their conductance $(115 \mathrm{pS}$ in $50 \mathrm{~mm} \mathrm{~K}$ glutamate) or by the size of the ions that could permeate the channel (including glutamate and HEPES, both of which are too large to permeate most conventional ion-conducting channels).

The number of channels observed ( $-3-15$ per fused vesicle) (see Fig. 1A) did not correlate with the number of ribosomes observed on the surface of each vesicle (100-200) (see Fig. 1B). One possibility was that these large channels were not involved in protein translocation. Alternatively, it was possible that when a channel was translocating a protein, its lumen would be occupied and thus occluded to the free flow of ions. Similar observations had been noted for ion channels. Many ion-channel blockers are actually large ions that only slowly transport through the channel, thereby blocking the ion currents that can be generated by smaller, faster-moving ions (Hille 1984). This alternative hypothesis could be tested by releasing nascent translocating chains from their ribosomes and observing whether this led to a large increase in the number of open channels we observed in the membrane. There are a number of ways of releasing nascent translocating chains. Agents that chelate magnesium, such as EDTA, dissociate the ribosomal subunits, thereby releasing the nascent chain still bound to its tRNA. However, we wanted to distinguish between effects of solely releasing the nascent chain and any effects of releasing the ribosomes from the membranes. Therefore, puromycin was used to release the nascent chains from the ribosomes.

Releasing nascent translocating peptides unblocks the protein-conducting channels. Puromycin is similar in structure to an amino acid bound to a tRNA. The peptidyl-transferase in the ribosome uses puromycin in place of an amino acid bound to a tRNA, which causes release of the peptidyl-puromycin from the ribosome (Traut and Monro 1964; Redman and Sabatini 1966; Blobel and Potter 1967; Monro and Marcker 1967). When the puromycin is added to solutions containing lower-than-physiological levels of salt $(<150 \mathrm{mM})$, the ribosome remains bound to the membranes after the puromycin reaction is complete. At, or above, physiological salt levels, the ribosomes release from the membranes (Adelman et al. 1973).

ER membranes were fused to a planar lipid bilayer and then $100 \mu \mathrm{M}$ puromycin was added to the solution bathing the cytoplasmic surface of the membrane (Simon and Blobel 1991). Almost immediately there was an increased conductance of $11 \mathrm{nS}$ (100 times the conductance of each of the large channels observed previously) (see Fig. 2A). The increased conductance could be specifically linked to release of the nascent translocating chains: (1) It was not observed when puromycin was added to a pure lipid membrane, (2) it was not observed when puromycin was added to a bilayer with $E R$ vesicles that had been stripped of ribosomes and mRNA, and (3) it was not observed when puromycin was added to the face of the membrane that correlated with the lumen of the ER (Simon and Blobel 1991).

The previous experiment demonstrated the electrophysiological consequences of releasing large numbers of nascent chains from their ribosomes with 100 $\mu \mathrm{M}$ puromycin. To observe the effects of releasing individual nascent chains, the concentration of puromycin was lowered to $0.3 \mu \mathrm{M}$. Shortly after addition of puromycin, there were step increases in the conductance of the membrane of $220 \mathrm{pS}$ (Fig. 2B, asterisk). These channels were large: They were permeable not only to 

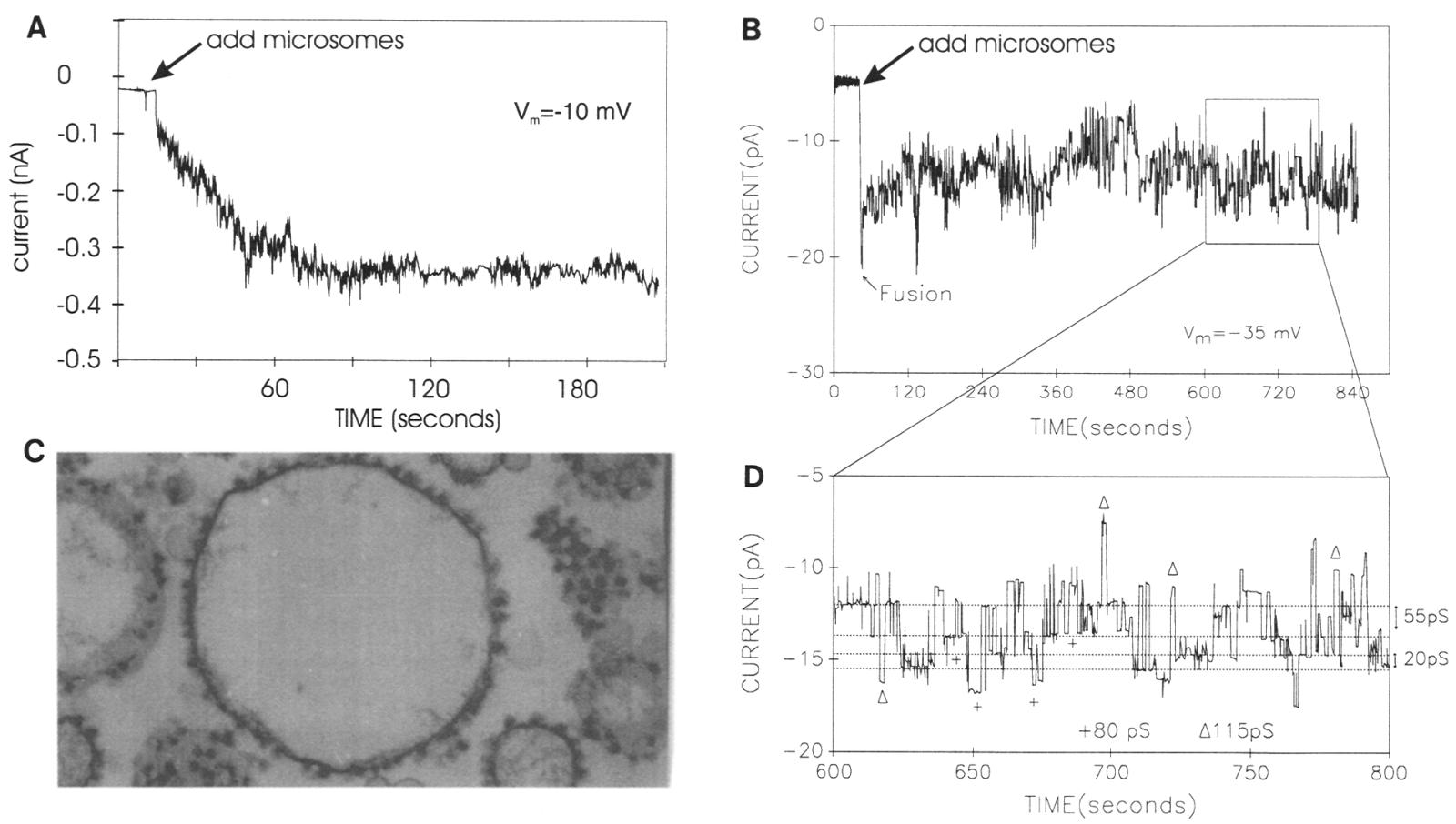

Figure 1. ER vesicles contain large aqueous channels. $(A)$ The conductance increase in response to fusing large numbers of ER vesicles to a planar lipid bilayer (Simon et al. 1989). (B) The fusion of individual ER vesicles to the bilayer can be observed if the vesicle concentration is substantially reduced (Simon et al. 1989). $(C)$ Electron micrograph of ER vesicles showing the ribosomes studding the surface. $(D)$ The time course of the record in $B$ was expanded to show individual channels (Simon et al. 1989).

$\mathrm{KCl}(\mathrm{g}=220 \mathrm{pS}$ at $50 \mathrm{~mm}$ ), but also to K-glutamate ( $\mathrm{g}$ $=180 \mathrm{pS}$ at $50 \mathrm{~mm}$ ) and to K-HEPES. The conductance of these channels was also unusually large. This can be best observed by contrasting the large 220 pS puromycin-evoked steps to the small channels marked with an arrowhead. The ER is the site of synthesis of plasma membrane proteins, including ion channels. The arrowheads mark conventional chloride-conducting channels that have been characterized by their ionic selectivity. Their presence dramatizes the difference in size between most conventional ion channels and these large presumptive protein-conducting channels.

To examine if the presence of ribosomes had any effect on these channels, the bathing salt concentration was raised to physiological levels and above to displace the ribosomes. Raising the salt concentration in small increments from $50 \mathrm{mM}$ initially had the effect of raising the conductance of each of the channels in the membrane. However, as the concentration reached 150 $\mathrm{mM}$ and above, the large puromycin-evoked channels closed (Fig. 2C). In contrast, all of the other smaller, ion-conducting channels increased in size as the concentrations of ions in the bathing media increased.

Signal sequences are ligands to open the proteinconducting channels. If these channels were closing at the termination of protein translocation, they would have to reopen at the initiation of protein translocation. What are the necessary and sufficient conditions to open these channels? In the ER, the translocation of proteins across the membrane is tightly coupled to the translation of proteins on the ribosome. We wanted to distinguish between potential roles for the ribosome in translation in contrast to its role in protein synthesis. Thus, we shifted our examination of the requirements for opening these channels to the plasma membrane of Escherichia coli. The translocation processes between the prokaryotic plasma membrane and eukaryotic ER are quite conserved: Eukaryotic proteins with signal sequences that target the ER when expressed in E. coli are properly targeted to and translocated across the plasma membrane, and vice versa. Activation of the translocation channels could be triggered by the signal sequence, the signal recognition particle (SRP, which binds the signal sequence), the ribosome, activation of the SRP receptor, or any combination of the above. Our strategy was to purify or synthesize each of these (either alone or in combination) and to test their effects on translocation-competent membranes.

Very few channels were observed upon fusing plasma membrane vesicles from $E$. coli to a planar lipid bilayer (Simon and Blobel 1992). This was expected, since the plasma membrane of $E$. coli is, in general, very impermeant. However, upon addition of $200 \mathrm{nM}$ signal peptide from the LamB protein, there was a dramatic increase of the conductance of the membrane (Fig. 3A). This increased conductance was a specific interaction of the signal peptides with the $E$. coli plasma membrane and not with the bilayer lipids. It was not observed when even a 1000 -fold higher concentration 
A

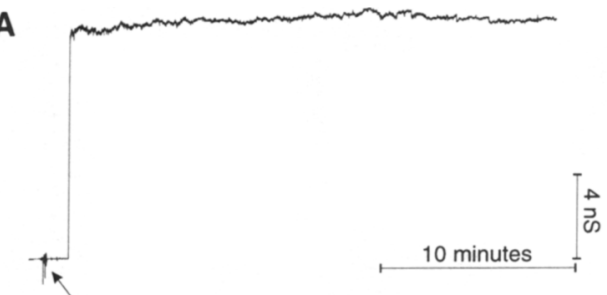

Add $100 \mu \mathrm{M}$ puromycin

B
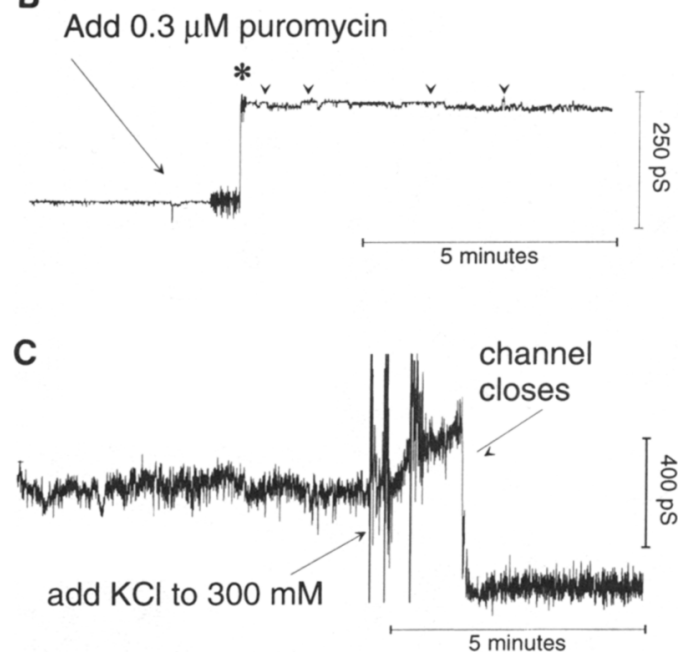

Figure 2. Releasing nascent chains reveals many large aqueous channels. $(A)$ ER vesicles were fused to a planar lipid bilayer. After addition of $100 \mu \mathrm{M}$ puromycin to the solution bathing the cytoplasmic surface of the ER membrane, there is a large increase in membrane conductance (Simon and Blobel 1991). (B) If a much lower concentration of puromycin is added, $0.3 \mu \mathrm{M}$, then discrete jumps are observed in the membrane conductance of $220 \mathrm{pS}$ (in $50 \mathrm{mM} \mathrm{KCl}$ ) (Simon and Blobel 1991). (C) The concentration of $\mathrm{KCl}$ bathing the membrane was increased in 50-mM steps. After each addition of $\mathrm{KCl}$, there was an increase in the conductance of all of the channels in the membrane. However, after the salt concentration rose to approximately physiological concentrations, and above, the large puromycin-evoked channels (and only these channels) closed (Simon and Blobel 1991). (Reprinted, with permission, from Simon and Blobel 1991 [copyright Cell Press].)

of signal peptide $(200 \mu \mathrm{M})$ was added to a bilayer made of $E$. coli lipids (Simon and Blobel 1992).

We next wanted to characterize the microscopic characteristics of the signal peptide-evoked membrane conductances in the $E$. coli membrane. When signal peptides were added at a substantially lowered concentration of $200 \mathrm{pm}$ (roughly the equivalent of one signal peptide for every eight $E$. coli volumes), a new channel was observed of $220 \mathrm{pS}$ (channel marked with an asterisk in Fig. 3B) that was not seen prior to the addition of the signal peptide. Together, these results demonstrate that large transmembrane aqueous channels can be observed in conjunction with the initiation
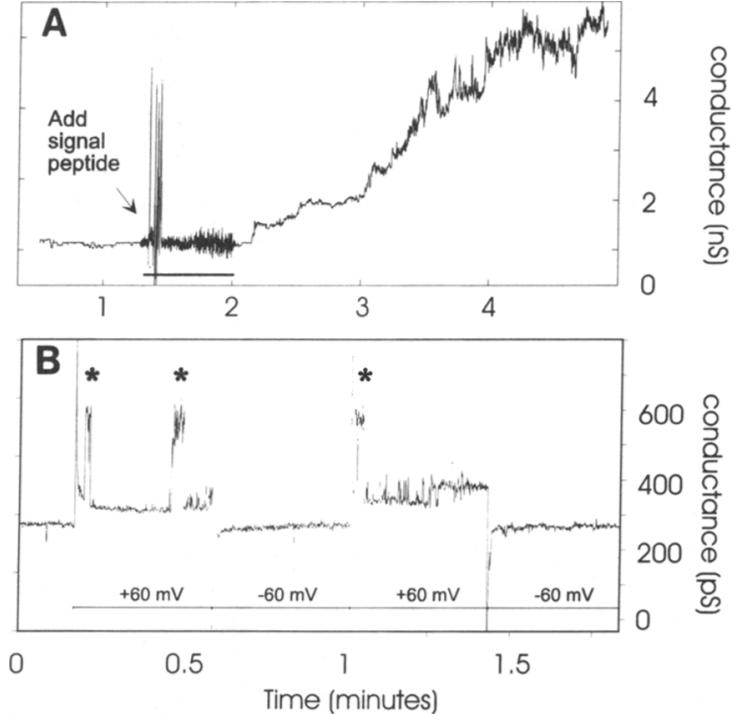

Figure 3. Signal peptides open large aqueous channels in translocation-competent membranes. $(A)$ Vesicles of $E$. coli plasma membrane were fused to a planar lipid bilayer. Upon addition of $200 \mathrm{nM}$ signal sequence from LamB to the solution bathing the cytoplasmic surface, there was a large increase in the conductance of the membrane (Simon and Blobel 1992). $(B)$ When the concentration of signal peptide was reduced to $200 \mathrm{pM}$, individual channels of $220 \mathrm{pS}$ could be observed (in $50 \mathrm{mM} \mathrm{KCl}$ ) (Simon and Blobel 1992). (Reprinted, with permission, from Simon and Blobel 1992 [copyright Cell Press].)

of protein translocation (upon addition of signal sequence) and at the end of protein translocation (during release of the nascent chain of the ribosome).

\section{What Moves the Proteins Across?}

If proteins are sitting in the middle of a transmembrane aqueous protein-conducting channel, then an important question is, What is responsible for moving the protein to the trans side (opposite the side of protein synthesis) of the membrane?

There are two potential mechanisms that could move the protein. There could be a motor in the membrane that moves the protein across. Almost any protein will translocate across the ER membrane if it is given a signal sequence. Therefore, this motor would have to be nonspecific and be able to recognize and bind any peptide sequence that is within the channel. The motor would have to bind tightly enough to pull the nascent translocating chain, yet bind quickly and reversibly enough to move the protein across the membrane. Finally, the motor would require a yet-to-becharacterized mechanism for transducing the chemical energy of ATP into molecular movement.

Alternatively, the protein, like ions, may be moving across the membrane from thermal fluctuations (Fig. 4A). How could this account for the net vectorial transport across the membrane? A number of factors 

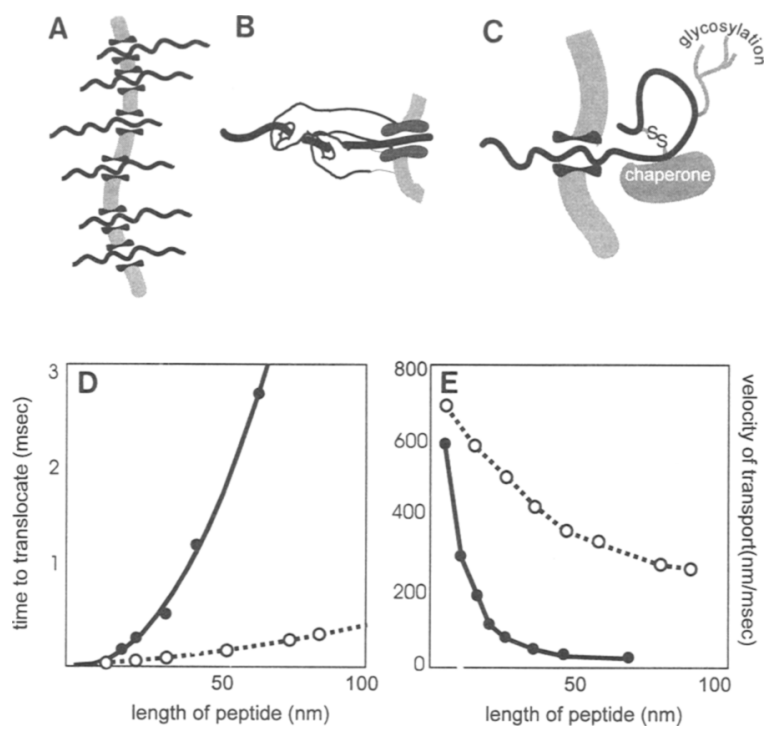

Figure 4. Models for protein transport. $(A)$ Illustration of a potential path of an unfolded protein fluctuating from thermal energy in a protein-conducting channel. (B) Model for a membrane-bound motor for protein movement. $(C)$ Model for a thermal ratchet which moves proteins through a combination of thermal fluctuations and ratcheting the protein on one side of the membrane. $(D)$ Predicted time course for a protein to translocate across the membrane increases with its length. Solid circles show the predicted time course assuming that the protein could be ratcheted at only one site. Open circles show the predicted time course if there are multiple ratcheting sites at even intervals. The symbols are the result of a numerical simulation and the solid lines are from an analytical solution (Simon et al. 1992). (E) Predicted velocity of transport assuming that there is either a single ratcheting site (filled circles) or multiple ratcheting sites (open circles) (Simon et al. 1992).

that can effect the ratcheting of the nascent translocating chain on the trans side of the membrane include folding, binding, and modification (Fig. 4C). Translocating proteins, as soon as they reach across the membrane, are modified by a variety of enzymatic activities: The signal sequence is cleaved, which affects folding of the nascent chain; the oligosaccharyltransferase adds a group of 14 sugars; disulfide bonds are formed; chaperones bind to nascent polypeptides translocating the ER, mitochondria, and chloroplast. If, for example, the energetics of the sugar-peptide bond are greater than the energetics of thermal fluctuations, the nascent chain will not be able to back-fluctuate out of the channel (Simon et al. 1992). Folding of the nascent translocating protein may also contribute to ratcheting of the protein on the trans side of the membrane. At the ER, proteins translocate as they are synthesized. Their first chance to fold is in the lumen of the ER. If the energetics of folding are comparable, or greater, than the energetics of thermal fluctuations, then the proteins will move across. Indeed, at the ER, the presence of a ribosome bound on the cytoplasmic side together with freedom for the chain to fold on the luminal side should be sufficient to ensure vectoriality. Numerous periplasmic bacterial proteins fold more tightly after their signal sequences are cleaved. Thus, they woutd fold more tightly after translocating, which would ensure vectoriality. The $\mathrm{pI}$ of mitochondrial proteins is 1.5 more basic than that of cytosolic proteins (Hartmann and Christen 1991). The alkaline pH of the mitochondria may induce more stable folding relative to the $\mathrm{pH}$ of the cytosol. Any or all of these mechanisms may contribute to ratcheting the nascent chain to the trans side of the membrane.

Thermal fluctuations, together with a ratchet, may contribute to moving a protein across the membrane. However, can it move a protein quickly enough to account for the kinetics of protein translocation? (Biology depends on kinetics.) This was tested by modeling, both numerically and analytically, the movement of the nascent translocating chain using standard approaches used for polymer dynamics (Simon et al. 1992). Both approaches predict that a thermal-driven fluctuation, coupled with a ratchet, could move most proteins across the membrane in milliseconds-fast enough to account for observed transport rates (Fig. 4D, E).

There are a number of predictions made from this model. Perhaps most importantly, it predicts that the vectoriality of translocation is driven not by a membrane-bound motor, but by a chemical potential gradient caused by soluble components on the cis (protein-synthesizing) and trans sides of the membrane. Eliminating these chemical potential gradients should block translocation. In the ER, there have been a number of experimental tests of the thermal-ratchet hypothesis. It was already known that a conditional mutation in $\mathrm{BiP}$, one of the main chaperone proteins within the lumen of the ER, would block translocation (Vogel et al. 1990). This observation was strengthened by the demonstration that yeast (Brodsky et al. 1993) and mammalian (Nicchitta and Blobel 1993) ER vesicles missing their luminal contents could initiate translocation, as assayed by cleavage of the signal sequence by the luminal signal peptidase, but could not import proteins.

Similar conclusions were drawn from the demonstration that short proteins, produced by truncated transcripts, could be shown to initiate translocation (as assayed by signal peptide cleavage) but would move retrograde out of the vesicles. The introduction of a glycosylation site into the amino-terminal end of the protein restored net transport (Ooi and Weiss 1992). Recently, immunologists studying the ER-resident peptide transporter (TAP1/TAP2) have taken advantage of this to engineer latent glycosylation sites into their peptides (see Jackson et al.; Ploegh; Sadasivan et al.; all this volume). This way, any peptide that is transported by the TAP will be ratcheted inside, allowing the measurement of a net TAP-dependent transport. Likewise, in the mitochondria there have been a number of tests of the thermal-ratchet hypothesis (Rassow et al. 1994; Schneider et al. 1994; 
Stuart et al. 1994; Ungermann et al. 1994). Nascent translocating chains have been shown to fluctuate back and forth in the translocation pore (Ungermann et al. 1994). Luminal chaperone proteins, which are transiently associated with the inside surface of the mitochondrial membrane, upon interaction and binding to a translocating chain, dissociate from the membrane. Thus, the luminal proteins appear to be bound to a translocation channel and are primed to bind to translocating chains, thereby ratcheting them on the inside. Similarly, it has recently been shown that chaperone proteins of the chloroplast are bound to nascent translocating chains (Schnell et al. 1994).

One experimental result has been suggested to be inconsistent with a thermal ratchet for translocation across the mitochondria. It was demonstrated that cytochrome $b_{2}$ cannot be imported into mitochondria unless either there is ATP in the mitochondria matrix, or if a relatively tightly folded domain of the cytochrome $b_{2}$ is destabilized by a mutation (Glick et al. 1993). This was interpreted to mean that a matrix ATP-dependent pump is unfolding the protein by pulling the nascent chain across the membrane (Glick 1995). An alternative explanation is that the folded domain can undergo small thermal fluctuations of structure that allow it to partially move across the membrane. With each advance across the membrane, the binding of chaperones is needed to ratchet it in the mitochondria. This could explain the ATP dependence. Most translocated proteins are unfolded on the cytoplasmic side and need only a single ratcheting event inside the mitochondria to ensure that they will move inside. Addition of ATP is needed to recycle and remove the chaperone from the nascent chain. For the short term, the ATP already bound to the chaperones should be sufficient for import. However, the cytochrome $b_{2}$ would require many repeated bindings of chaperones to drive unfolding. Thus, ATP will be needed for recycling the chaperones.

To distinguish between the two models, the critical question is whether ATP hydrolysis is required to stabilize a protein movement that has occurred from thermal fluctuation (in which case each of the energetic barriers should be $<2 \mathrm{kT}$ ) or whether ATP hydrolysis is being used to unfold a protein past an energetic barrier of $>12 \mathrm{kT}$. In the latter case, the energy of ATP is being directly coupled to protein unfolding. All proteins undergo fairly substantial conformation changes at thermal equilibrium (which is why we can observe "spontaneous" openings and closings even in large proteins such as the sodium channel). To evaluate this experiment further, it is necessary to know if unfolding of the protein occurs through eight steps, each with a barrier of $2 \mathrm{kT}$, or if there is a single step with a barrier of $16 \mathrm{kT}$. Since the vast majority (even of mitochondrial proteins) do not have to encounter this problem, it is not unreasonable to assume that a thermal ratchet is the norm for moving proteins across membranes. This is perhaps even more so in situ, where most mitochondrial proteins translocate either cotranslationally or at least before they have a chance to fold. In the particular artificial situation of a protein that has been allowed to fold prior to an in vitro posttranslational translocation, we may be observing an emergency response on the part of the mitochondria. This reaction may be elicited in response to proteins that have clogged its lifeline to the outside world. Thus, if there is an ATP-dependent pump pulling the cytochrome $b_{2}$, it may be an important reaction, but not relevant to the normal mechanisms for protein translocation.

\section{How Are Membrane Proteins Incorporated into the Bilayer?}

In many ways, the behavior of these proteinconducting channels resembles that of conventional ion-conducting channels (see Table 1). However, there is one very distinct difference between the two: Ions transport only perpendicularly across the membrane. In contrast, whereas secretory proteins translocate only perpendicularly to the membrane, latent membrane proteins also move in a second dimension: parallel to the plane of the membrane to integrate into the lipid bilayer.

Do nascent membrane and secretory proteins utilize the same translocation machinery? There is considerable biochemical evidence supporting common steps in the translocation steps. Both secretory and membrane proteins require SRP to initiate translocation (Anderson et al. 1982), and they compete with each other for translocation (Lingappa et al. 1979). Some signal sequences can function as transmembrane domains and some transmembrane domains can function as SRP. dependent signal sequences. Both secretory and membrane proteins can be cross-linked to sec61p-an ERresident membrane protein that has been implicated as a key player in translocation based on genetic selections and biochemical studies (Deshaies and Schekman

Table 1. Comparison of Protein-conducting and Ion-conducting Channels

\begin{tabular}{|c|c|}
\hline Protein-conducting channels & Ion-conducting channels \\
\hline $\begin{array}{l}\text { Proteins move by thermal } \\
\text { fluctuations through an } \\
\text { open aqueous pore. A } \\
\text { chemical potential gradient } \\
\text { determines the direction } \\
\text { of net transport. }\end{array}$ & $\begin{array}{l}\text { Ions move by thermal } \\
\text { fluctuations through } \\
\text { an open aqueous pore. A } \\
\text { chemical potential } \\
\text { gradient determines the } \\
\text { direction of net transport. }\end{array}$ \\
\hline $\begin{array}{l}\text { Translocating chains block } \\
\text { current when they are } \\
\text { occupying the lumen of } \\
\text { the pore. }\end{array}$ & $\begin{array}{l}\text { Peptides at the amino } \\
\text { terminus of the } \mathrm{K}^{+} \\
\text {channel block current } \\
\text { (inactivate the channel) }\end{array}$ \\
\hline $\begin{array}{l}\text { Signal sequence is the } \\
\text { ligand to open the channel. }\end{array}$ & $\begin{array}{l}\text { when inserted into the pore. } \\
\text { Neurotransmitters or } \\
\text { hormones can open the } \\
\text { channel upon binding to a } \\
\text { receptor. }\end{array}$ \\
\hline
\end{tabular}


1987; Rothblatt et al. 1989; Görlich et al. 1992; Stirling et al. 1992; High et al. 1993; Oliver et al. 1995). Proteins that have a single transmembrane domain often have, like secretory proteins, a cleaved aminoterminal signal sequence (Lingappa et al. 1978). Nature has provided some strong evidence for shared mechanisms for translocation: Some immunoglobins are synthesized in both a membrane-bound and secretory form (McCune et al. 1980). All of them are generated from mRNA that come from a common transcript which is differently spliced to have, or delete, a carboxy-terminal transmembrane segment. Protein synthesis is initiated at the amino terminus. The amino terminus of these proteins has fully translocated before the ribosomes reach the coding region for the carboxyterminal transmembrane domain. All of these indicate that, at a minimum, membrane and secretory proteins have many shared steps in the translocation.

What is the environment for a membrane protein during translocation? We have been addressing two questions in collaboration with Dr. Melvin Schindler of Michigan State University. First, do membrane proteins, like secretory proteins, also translocate through an aqueous protein-conducting channel? Second, are proteins translocating through the aqueous proteinconducting channel exposed to, or completely shielded from, the lipid bilayer? The experimental question we asked was whether a nascent translocating transmembrane protein that was still attached to its tRNA and biosynthetic ribosome was exposed to the lipid bilayer. Exposure to the lipid bilayer was assayed by the ability to label the nascent translocating protein by a hydrophobic photoactivatable label, 3-(trifluoro-methyl)-3(m-[ $\left.{ }^{125} \mathrm{I}\right]$ iodophenyl)diazirine (TID), that was preloaded into the bilayer (Brunner and Semenza 1981).

Our model membrane protein was the vesicular stomatitis virus glycoprotein (VSV-G). The mature full-length VSV-G has a single transmembrane segment near its carboxyl terminus. TID has been used to label the transmembrane segments of many different proteins, and it will nonspecifically label almost every amino acid.

We wanted to assay whether VSV-G protein, during biosynthesis, could be labeled by TID from within the lipid bilayer. To trap VSV-G protein at a specific biosynthetic stage, its mRNA was transcribed from a plasmid that was linearized shortly after the region that codes for the transmembrane segment. This produces a truncated mRNA that lacks a termination codon (see Fig. 5). During translation, the ribosome reaches the end of the mRNA and halts. The nascent polypeptide chain remains attached via its carboxy-terminal amino acid and cognate tRNA to its biosynthetic ribosome. To specifically assay the TID labeling of only those translation intermediates still attached to their biosynthetic tRNA and ribosome, CTAB was used, after TID labeling, to specifically precipitate all tRNAbound translation products.

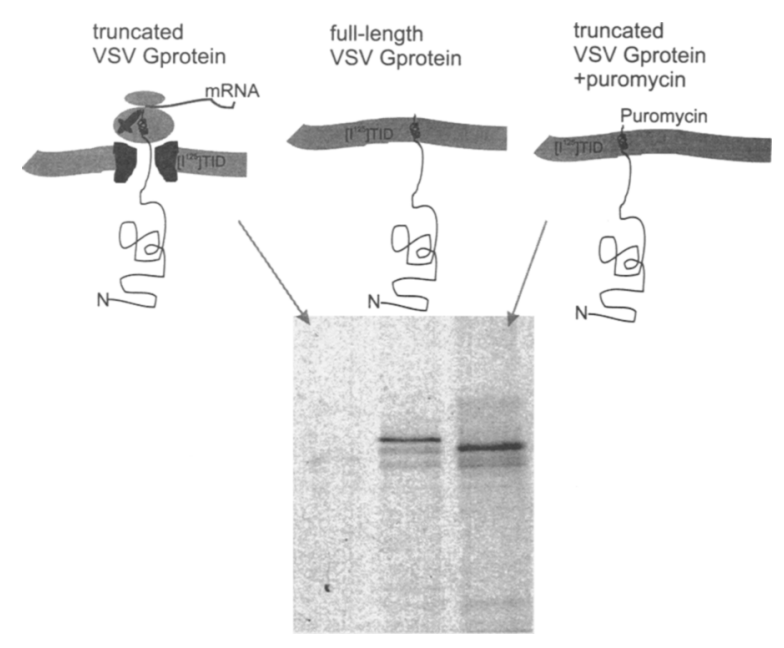

Figure 5. Translocating chains are not exposed to the lipid bilayer. mRNA for VSV-G protein was synthesized in the presence of ER vesicles which were loaded with the small hydrophobic photoactivatable probe TID. However, this mRNA was truncated shortly after the coding region for single transmembrane domain of the protein. This leaves the nascent polypeptide attached to its tRNA and ribosome. The preparation is split in two and one half is treated with puromycin, which releases the nascent polypeptide thereby allowing it to integrate into the lipid bilayer. The TID was then photoactivated in both reactions. The truncated tRNAattached translocation intermediate form of the VSV-G protein showed no apparent labeling with TID (left lane). In contrast, the full-length VSV-G protein (middle lane) and the translocation intermediate that had been released from its ribosome (right lane) both were labeled with TID. This indicates that there was sufficient VSV-G protein in the translation reaction to show TID labeling. Therefore, the lack of label on the intermediates in translocation shows that during translocation they cannot be accessed from within the hydrocarbon core of the bilayer.

We had previously tried to label nascent translocating secretory proteins, such as preprolactin and prepro- $\alpha$-factor, and could not observe any TID label of the translocating chain. We were concerned that we were synthesizing such small amounts of the nascent peptide, and TID labeling was so inefficient, that the TID label was not strong enough to be observed. Thus, it was very important to have a positive control for this experiment. One advantage of testing for TID labeling of a nascent membrane protein was that we could split our translation mix in half, and treat one half with puromycin to release the nascent translocating chains from the ribosomes. The reaction treated with puromycin quickly integrates into the bilayer. If there were enough nascent protein to label with TID, then we should have a positive signal from the VSV-G that had been released from the ribosome with puromycin (Fig. 5). The key issue would then be whether TID labeling was observed on the tRNA-bound nascent chain.

VSV-G was translated from truncated mRNA in the presence of ER vesicles that had been loaded with TID. The TID was then photoactivated. The ER 
vesicles were then harvested and all tRNA-bound peptides were precipitated. Nascent VSV-G was immunoprecipitated from this pellet of tRNA-conjugated nascent peptides.

TID labeling was not observed on the nascent translocating VSV-G (Fig. 5, left lane). In contrast, TID labeling was observed in both of the positive controls: the full-length VSV-G (Fig. 5, middle lane) and the nascent translocating chains that had been released from their ribosomes prior to activation of TID (Fig. 5, right lane). These results indicate that the nascent translocating membrane protein is not moving through an environment exposed to the phospholipids of the bilayer. They are fully consistent with the observation in $E$. coli that during translocation, translocating proteins can be cross-linked to the proteins SecY and SecA, but cannot be cross-linked to lipid (Joly and Wickner 1993).

Polytopic membrane proteins, those with multiple transmembrane domains, do not have cleaved aminoterminal signal sequences. They present more complex problems when it comes to integration of their transmembrane domains. To characterize the process of integration of proteins with multiple transmembrane segments (TMS) into the bilayer, we have asked two experimental questions. First, does each TMS translocate across the membrane as it emerges from the ribosome? Second, does each TMS integrate into the lipid bilayer as it achieves a transmembrane orientation? We have been using as our experimental proteins the mammalian P-glycoprotein (mdr1) and in collaboration with Dr. Martin Friedlander of Scripps, we have been examining opsin and the rod $\mathrm{Na} / \mathrm{Ca}$ exchanger. Our results indicate that the nascent transmembrane domains of these proteins translocate across the membrane sequentially, as they emerge from the ribosome. However, the transmembrane segments do not integrate into the lipid bilayer when they are oriented across the membrane. This event is delayed long after, perhaps only until the entire membrane protein is synthesized and released from its biosynthetic ribosome. After the transmembrane segments traverse the membrane, but before they are integrated into the lipid bilayer, they are stabilized with the membrane. However, this stabilization is not through the hydrophobic transmembrane segments, but through salt-sensitive electrostatic interactions (S.M. Simon, in prep.).

\section{DISCUSSION}

\section{The Model}

Our results are strongly supportive of the following model for the translocation of secretory and membrane proteins. Translocation of proteins across the membrane is proposed to occur through transmembrane aqueous protein-conducting channels. These channels are closed; that is, they are not freely permeable to the flow of ions until the nascent signal sequence (which for polytopic membrane proteins is usually the first TMS) of the protein is presented to the channel. The signal sequence binds to the channel, potentially via its hydrophobic stretch and positively charged amino terminus (Simon and Blobel 1992). The channel then opens, followed by the rapid insertion of the nascent translocating chain into the lumen of the channel. Thus, although the channel is open and permissive to the transport of a nascent translocation chain, it is occluded and therefore not freely permeable to the flow of ions through the channel.

Appearance of a short stretch of the nascent chain on the trans (opposite) side is accompanied by modifications of the nascent chain (additions of sugars, formation of disulfide bonds). Thus, even though the nascent chain is fluctuating from thermal energy, any sites on the protein that have been modified on the trans side can no longer fluctuate back into the proteinconducting channel.

Secretory proteins will continue to move across the membrane until the ribosome reaches the termination codon in their mRNA. Then, in steps yet to be resolved, the nascent chain is released from the ribosome and the ribosome dissociates from the translocation site. Upon dissociation of the ribosome from the membrane, the protein-conducting channels close.

The biogenesis of membrane proteins follows a similar strategy with one exception. The latent transmembrane segments do not fully move across the membrane but stop partially across the membrane. The latent transmembrane segments, which function as signal sequences, are recognized and bind via ionic linkages to the protein-conducting channels, or chaperonelike proteins associated with the channels. They also do not immediately partition into the bilayer. They remain within an aqueous accessible compartment, allowing the transmembrane segments to fold and achieve their proper transmembrane topography until the entire protein is synthesized. Only after release of the protein from the ribosome do the protein-conducting channels close, allowing the nascent membrane protein to enter the lipid bilayer.

Evidence for an aqueous channel comes from both electrophysiological and biochemical experiments. Together they form a compelling case for protein-conducting channels. The electrophysiological experiments demonstrated that the signal sequence is sufficient to open large-transmembrane aqueous channels in translocation-competent membranes. Channels of similar conductance and permeability were observed when translocating proteins were preinaturely released from their ribosomes under conditions that maintain the ribosomes attached to the membrane. The biochemical data demonstrated that even latent transmembrane proteins remain within an aqueous compartment shielded from the phospholipids during translocation. Even a seven-transmembrane-segment protein that has been fully synthesized, short of its termination codon, is not integrated into the membrane. 
Both the biochemical and electrophysiological experiments strongly support a role for the ribosome, independent of its role in protein synthesis, in regulating protein translocation across the ER. After the proteinconducting channels were unblocked by releasing the nascent chains, the channels remained open and freely conductive to ions. It was only after the ribosomes were exposed to a physiological salt solution, which allows the ribosomes to dissociate, that the channels closed. During the synthesis of membrane proteins, the nascent chains did not embed in the bilayer, even after all of the transmembrane segments were synthesized. It was only upon release of the nascent chain from the ribosome that the latent membrane protein integrated into the membrane. This was true whether only a single transmembrane segment or all seven transmembrane segments of a protein had been synthesized. These results support a role for the ribosome in keeping nascent polypeptides in the translocation channel. This is consistent with the observation that nascent membrane proteins could be cross-linked to sec61 as long as they were still attached to their ribosome, even if 100 amino acids had been synthesized after the transmembrane domain (Thrift et al. 1991). Unfortunately, a better understanding of how the ribosome effects its actions may have to wait until there is better characterization of the interactions between ribosomes and the translocation machinery.

This model for translocation is based on a series of experiments that used nonphysiological treatments: opening the channels with signal peptides, releasing the nascent chains with puromycin, closing the channels with high salt, or extracting proteins with urea. These nonphysiological treatments were necessary for tricking the channels into exposing their presence. However, the results are consistent with the known physiology. Channels should not be open in situ. Opening the channels across the ER would dissipate gradients and metabolites across this organelle. Perhaps a small leakage through these channels at the initiation or termination of translocation is responsible for the leakage of calcium across the ER. If the ER calcium pumps are blocked with thapsigargin, the luminal calcium rapidly leaks out (Metz et al. 1992; Toescu et al. 1992). Similarly, an inappropriate opening of these channels in $E$. coli would be lethal. Indeed, this may be the mechanism of toxicity of a mutant hydrophilic signal peptide that depolarizes the $E$. coli membrane (Pollitt and Inouye 1988).

These results are also consistent with a number of other published results. Nascent translocation chains in the ER have been shown to be in an aqueous environment, as assayed by their sensitivity to urea extraction (Gilmore and Blobel 1985) and by the fluorescent signal of a probe attached to the translocating chain (Crowley et al. 1993). Addition of proteins (or peptides) competent for membrane translocation has been demonstrated to affect the electrical conductance of mitochondria (Henry et al. 1989; Thieffry et al. 1992;
Vallette et al. 1994) and chloroplasts (Bulychev et al. 1994). In $E$. coli, nascent translocating chains that were trapped across the membrane during translocation could not be cross-linked to lipids, again supporting the notion of a channel that is shielded from the lipid bilayer (Joly and Wickner 1993). The evidence that translocating chains in the ER and mitochondria are capable of fluctuations back and forth is also consistent with an aqueous channel for translocation (Ooi and Weiss 1992; Brodsky et al. 1993; Nicchitta and Blobel 1993; Ungermann et al. 1994).

\section{Advantages for the Cell}

Thermodynamics of transport. Such a mechanism for the biogenesis of proteins provides a number of significant benefits for cells. Many proteins that are fully translocated across the membrane have long stretches of hydrophobic amino acids. It would be energetically favorable for these stretches to embed into a lipid bilayer. Similarly, it would be highly energetically unfavorable to extract these domains out of the lipid. All nascent peptides are synthesized within an aqueous large channel in the ribosome (Yonath et al. 1987; Crowley et al. 1993). These peptides are moved through the water by thermal fluctuations. Once hydrophobic stretches are in water, no additional energy is needed to move them along. By translocating these chains through an aqueous channel shielded from lipid, no additional energy is needed to get the hydrophobic stretches across the membrane. If, on the other hand, these stretches translocated, even transiently, through the lipid, or through a mixed lipid/protein complex, it would require tremendous energy to extract these proteins to the trans side.

Life in the realm of thermal fluctuations. Proteins exist in a world dominated by thermal fluctuations. Most of the forces that concern us (inertia, gravity, and grants) are not particularly relevant to their world. Protein movement must be viewed from their perspective (Gamow 1973). Proteins are not run by gears, springs, or rubber bands. Thermal fluctuations are a dominant and driving force. Any reactions that can bias their effect will harness a powerful force. Proteins sitting in an aqueous channel will fluctuate as surely as any other polymers (DeGennes 1976, 1983). The known modifications that occur to translocating chains (binding of sugar groups, chaperones, formation of disulfide bonds, cleavage of signal sequences) are sufficient to bias the thermal fluctuation and drive directional protein translocation. The cell does not derive work from the thermal fluctuations (Feynman et al. 1963). The thermal fluctuations themselves are inherently unbiased. However, work is being generated from the ATP used in glycosylation, in the chaperonebinding cycle, or in maintaining the $\mathrm{pH}$ gradient across the mitochondria. Like ions, the proteins move across the membrane from thermal fluctuations with the work 
coming from a gradient of chemical potential across the membrane.

Integration of membrane proteins. For polytopic membrane proteins, there is a distinct advantage to not integrating into the lipid bilayer until the entire protein is synthesized. Charged amino acids are frequently found in transmembrane domains. These groups are stable with the bilayer because they are found to be salt-linked to other charged groups in other transmembrane domains. Holding the TMS from integrating into the lipid bilayer until all of the protein is synthesized allows these groups to find each other and bond. It also allows the different TMSs to fold into an appropriate conformation for integrating into the membrane. Perhaps this is why the latent TMSs are not stabilized across the membrane via their hydrophobic segments. By stabilizing these groups via ionic links outside of the membrane, the hydrophobic domains are free to interact and fold.

\section{Are Aqueous Pores a General Mechanism for Macromolecular Movement?}

The selective modification of membrane permeability is a major challenge for biological membranes. Aqueous channels have been demonstrated to be an important mechanism for the transport of ions across membranes. Our results strongly support a role for aqueous channels in the translocation of proteins across membranes. A similar mechanism may have evolved for the transport of other hydrophilic molecules such as amino acids (e.g., the histidine permease) or peptides (e.g., a-factor transport in yeast or peptide transport across the ER in antigen presentation). The observation that appropriate peptides move in both directions across the membrane is strongly suggestive of a transmembrane aqueous pore rather than a pump which flips the peptide from one side of the membrane to the other (see Jackson et al.; Ploegh; Sadasivan et al; all this volume). We already know that large aqueous channels are used for the transport of nucleic acids: RNA exits the nucleus through the nuclear pores (see articles on nuclear transport by Blobel; Michael et al.; Izaurralde et al.; Fabre et al.; Adam et al.; Görlich and Laskey; Moore and Blobel; Melchior et al.; all this volume) and both T4 and T5 phage have been demonstrated to inject their genetic material into bacteria through transmembrane aqueous channels (Boulanger and Letellier 1988, 1992; Guihard et al. 1993). Indeed, once filamentous phage replicate in bacteria, they are extruded across both bacterial membranes with few ill consequences for the viability of the cell or the integrity of the membrane. Perhaps it is not unreasonable to ask if even large macromolecular complexes, like phage, composed of DNA and proteins, are not also translocated across membranes through transmembrane aqueous channels.

The next few years should see rapid progress in at least two directions: first, in determining how general aqueous pores are for the transport of molecules across membranes. We may even find that "ion pumps" use transmembrane aqueous pathways for their transport. Second, as the state of the art advances in the reconstitution of membrane proteins, we should start seeing reconstitutions of protein translocation that are faithful enough in their rates and efficiency to allow detailed analyses of the structure-function relationship between the molecules that form the protein-conducting channels.

\section{ACKNOWLEDGMENTS}

I thank all of my collaborators, G. Blobel, A. Borel, M. Friedlander, G.F. Oster, C. Peskin, M. Schindler, J. Zimmerberg, and all of the members of my laboratory, for their time and thoughts. Special thanks to $\mathbf{M}$. Schindler for comments on the manuscript.

\section{REFERENCES}

Adelman, M.R., D.D. Sabatini, and G. Blobel. 1973. Ribosome-membrane interaction. Nondestructive disassembly of rat liver rough microsomes into ribosomal and membranous components. J. Cell Biol. 56: 206.

Anderson, D.J., P. Walter, and G. Blobel. 1982. Signal recognition protein is required for the integration of acetylcholine receptor $\delta$ subunit, a transmembrane glycoprotein into the endoplasmic reticulum membrane. J. Cell Biol. 93: 501.

Blobel, G. and B. Dobberstein. 1975. Transfer of proteins across membranes. II. Reconstitution of functional rough microsomes from heterologous components. J. Cell Biol. 67: 852 .

Blobel, G. and V.R. Potter. 1967. Studies on free and membrane-bound ribosomes in rat liver. II. Interaction of ribosomes and membranes. J. Mol. Biol. 26: 293.

Blobel, G. and D.D. Sabatini. 1971. Ribosome-membrane interaction in eukaryotic cells. In Biomembranes 2 (ed. L.A. Manson), p. 193. Plenum Press, New York.

Boulanger, P. and L. Letellier. 1988. Characterization of ion channels involved in the penetration of phage T4 DNA into Escherichia coli cells. J. Biol. Chem. 263: 9767.

- 1992 . Ion channels are likely to be involved in the two steps of phage T5 DNA penetration into Escherichia coli cells. J. Biol. Chem. 267: 3168.

Brodsky, J.L., S. Hamamoto, D. Feldheim, and R. Schekman. 1993. Recorstitution of protein translocation from solubilized yeast membranes reveals topologically distinct roles for BiP and cytosolic Hsc70. J. Cell Biol. 120: 95.

Brunner, J. and G. Semenza. 1981. Selective labeling of the hydrophobic core of membranes with 3-(trifluoromethyl)$3-\left(\mathrm{m}-\left[{ }^{125} \mathrm{I}\right]\right.$ iodophenyl $)$ diazirine, a carbene-generating reagent. Biochemistry 20: 7174 .

Bulychev, A., M. Pilon, H. Dassen, R. Van 't Hof, W. Vredenberg, and B. De Kruijff. 1994. Precursor-mediated opening of translocation pores in chloroplast envelopes. FEBS Lett. 356: 204.

Crowley, K.S., G.D. Reinhart, and A.E. Johnson. 1993. The signal sequence moves through a ribosomal tunnel into a noncytoplasmic aqueous environment at the ER membrane early in translocation. Cell 73: 1101.

DeGennes, P.G. 1976. Dynamics of entangled polymer solu- 
tions. I. The rouse model. Macromolecules 9: 587. . 1983. Reptation d'une chaine heterogene. J. Physique Lett. 44: 1225.

Deshaies, R.J. and R. Schekman. 1987. A yeast mutant defective at an early stage in import of secretory protein precursors into the endoplasmic reticulum. J. Cell Biol. 105: 633.

Feynman, R., R. Leighton, and M. Sands. 1963. The Feynman lectures on physics. Addison-Wesley, Reading, Massachusetts.

Gamow, G. 1973. Mr. Tompkins in paperback. Cambridge University Press, London, United Kingdom.

Gilmore, R. and G. Blobel. 1985. Translocation of secretory proteins across the microsomal membrane occurs through an environment accessible to aqueous perturbants. Cell 42: 497.

Glick, B.S. 1995. Can Hsp70 proteins act as force-generating motors? Cell 80: 11.

Glick, B.S., C. Wachter, G.A. Reid, and G. Schatz. 1993. Import of cytochrome b2 to the mitochondrial intermembrane space: The tightly folded heme-binding domain makes import dependent upon matrix ATP. Protein Sci. 2: 1901.

Görlich, D., S. Prehn, E. Hartmann, K.-U. Kalies, and T.A. Rapoport. 1992. A mammalian homolog of SEC61p and SECYp is associated with ribosomes and nascent polypeptides during translocation. Cell 71: 489.

Guihard, G., H. Bénédetti, M. Besnard, and L. Letellier. 1993. Phosphate efflux through the channels formed by colicins and phage T5 in Escherichia coli cells is responsible for the fall in cytoplasmic ATP. J. Biol. Chem. 268: 17775.

Hartmann, C. and P. Christen. 1991. Mitochondrial protein charge. Nature 352: 762.

Henry, J.P., J.-F. Chich, D. Goldschmidt, and M. Thieffry. 1989. Blockade of a mitochondrial cationic channel by an addressing peptide: An electrophysiological study. $J$. Membr. Biol. 112: 139.

High, S., S.S.L. Andersen, D. Görlich, E. Hartmann, S. Prehn, T.A. Rapoport, and B. Dobberstein. 1993. Sec61p is adjacent to nascent type I and type II signal-anchor proteins during their membrane insertion. J. Cell Biol. 121: 743.

Hille, B. 1984. Ionic channels of excitable membranes. Sinauer, Sunderland, Massachusetts.

Joly, J.C. and W. Wickner. 1993. The SecA and SecY subunits of translocase are the nearest neighbors of a translocating preprotein, shielding it from phospholipids. EMBO J. 12; 255.

Lingappa, V.R., F.N. Katz, H.F. Lodish, and G. Blobel. 1978. A signal sequence for the insertion of a transmembrane glycoprotein. Similarities to the signals of secretory proteins in primary structure and function. J. Biol. Chem. 253: 8667.

Lingappa, V.R., B.A. Cunningham, S.M. Jazwinski, T.P. Hopp, G. Blobel, and G.M. Edelman. 1979. Cell-free synthesis and segregation of $\beta 2$-microglobulin. Proc. Natl. Acad. Sci. 76: 3651 .

McCune, J.M., V.R. Lingappa, S.M. Fu, G. Blobel, and H.G. Kunkel, 1980. Biogenesis of membrane-bound and secreted immunoglobulins. I. Two distinct translation products of human $\mu$-chain, with identical N-termini and different C-termini. J, Exp. Med. 152: 463.

Metz, D.C., R.J. Patto, J.E. Mrozinski, Jr., R.T. Jensen, R.J. Turner, and J.D. Gardner. 1992. Thapsigargin defines the roles of cellular calcium in secretagogue-stimulated enzyme secretion from pancreatic acini. J. Biol. Chem. 267: 20620.
Monro, R.E. and K.A. Marcker. 1967. Ribosome-catalysed reaction of puromycin with a formylmethionine-containing oligonucleotide. J. Mol. Biol. 25: 347.

Mueller, P., D.O. Rudin, H.T. Tien, and W.C. Wescott. 1963. Methods for the formation of single bimolecular lipid membranes in aqueous solution. J. Phys. Chem. 67: 534.

Nicchitta, C.V. and G. Blobel. 1993. Lumenal proteins of the mammalian endoplasmic reticulum are required to complete protein translocation. Cell 73: 989.

Niles, W.D. and' F.S. Cohen, 1987. Video fluorescence microscopy studies of phospholipid vesicle fusion with a planar phospholipid membrane. Nature of membrane-membrane interactions and detection of release of contents. J. Gen. Physiol. 90: 703.

Oliver, J., B. Jungnickel, D. Görlich, T. Rapoport, and S. High. 1995. The Sec61 complex is essential for the insertion of proteins into the membrane of the endoplasmic reticulum. FEBS Lett. 362: 126.

Ooi, C.E. and J. Weiss. 1992. Bidirectional movement of a nascent polypeptide across microsomal membranes reveals requirements for vectorial translocation of proteins. Cell 71: 87.

Pollitt, N.S. and M. Inouye. 1988. Synthesis of an Escherichia coli protein carrying a signal peptide mutation causes depolarization of the cytoplasmic membrane potential. $J$. Bacteriol. 170: 2051.

Rassow, J., A.C. Maarse, E. Krainer, M. Kübrich, H. Müller, M. Meijer, E.A. Craig, and N. Pfanner. 1994. Mitochondrial protein import: Biochemical and genetic evidence for interaction of matrix hsp70 and the inner membrane protein MIM44. J. Cell Biol. 127: 1547.

Redman, C.M. and D.D. Sabatini. 1966. Vectorial discharge of peptides released by puromycin from attached ribosomes. Proc. Natl. Acad. Sci. 56: 608.

Schneider, H.-C., J. Berthold, M.F. Bauer, K. Dietmeier, B. Guiard, M. Brunner, and W. Neupert. 1994. Mitochondrial Hsp70/MIM44 complex facilitates protein import. Nature 371: 768 .

Schnell, D.J., F. Kessler, and G. Blobel. 1994. Isolation of components of the chloroplast protein import machinery. Science 266: 1007.

Simon, S.M. and G. Blobel. 1991. A protein-conducting channel in the endoplasmic reticulum. Cell 65: 371 .

. 1992. Signal peptides open protein-conducting channels in E. coli. Cell 69: 677.

Simon, S.M., G. Blobel, and J. Zimmerberg. 1989. Large aqueous channels in membrane vesicles derived from the rough endoplasmic reticulum of canine pancreas or the plasma membrane of Escherichia coli. Proc. Natl. Acad. Sci. 86: 6176.

Simon, S.M., C.S. Peskin, and G.F. Oster. 1992. What drives the translocation of proteins? Proc. Natl. Acad. Sci. 89: 3770.

Stirling, C.J., J. Rothblatt, M. Hosobuchi, R. Deshaies, and R. Schekman. 1992. Protein translocation mutants defective in the insertion of integral membrane proteins into the endoplasmic reticulum. Mol. Biol. Cell 3: 129.

Stuart, R.A., A. Gruhler, I. Van der Klei, B. Guiard, H. Koll, and W. Neupert. 1994. The requirement of matrix ATP for the import of precursor proteins into the mitochondrial matrix and intermembrane space. Eur. J. Biochem. 220: 9.

Thieffry, M., J. Neyton, M. Pelleschi, F. Fèvre, and J.-P. Henry. 1992. Properties of the mitochondrial peptidesensitive cationic channel studied in planar bilayers and patches of giant liposomes. Biophys. J. 63: 333. 
Thrift, R.N., D.W. Andrews, P. Walter, and A.E. Johnson. 1991. A nascent membrane protein is located adjacent to ER membrane proteins throughout its integration and translation. J. Cell Biol. 112: 809.

Toescu, E.C., A.M. Lawrie, O.H. Petersen, and D.V. Gallacher. 1992. Spatial and temporal distribution of agonistevoked cytoplasmic $\mathrm{Ca}^{2+}$ signals in exocrine acinar cells analysed by digital image microscopy. EMBO J. 11: 1623.

Traut, R.R. and R.E. Monro. 1964. The puromycin reaction and its relation to protein synthesis. J. Mol. Biol. 10: 63 .

Ungermann, C., W. Neupert, and D.M. Cyr. 1994. The role of Hsp70 in conferring unidirectionality on protein translocation into mitochondria. Science 266: 1250.

Vallette, F.M., P. Juin, M. Pelleschi, and J.-P. Henry. 1994. Basic peptides can be imported into yeast mitochondria by two distinct targeting pathways. Involvement of the peptide-sensitive channel of the outer membrane. J. Biol. Chem. 269: 13367.

Vogel, J.P., L.M. Misra, and M.D. Rose. 1990. Loss of
BiP/GRP78 function blocks translocation of secretory proteins in yeast. J. Cell Biol. 110: 1885 .

Walter, P. and G. Blobel. 1983. Preparation of microsomal membranes for cotranslational protein translocation. Methods Enzymol. 96: 84.

Walter, P. and V.R. Lingappa. 1986. Mechanism of protein translocation across the endoplasmic reticulum membrane. Annu. Rev. Cell Biol. 2: 499.

Yonath, A., K.R. Leonard, and H.G. Wittmann. 1987. A tun. nel in the large ribosomal subunit revealed by threedimensional image reconstruction. Science 236: 813.

Zagotta, W.N., T. Hoshi, and R.W. Aldrich. 1990. Restoration of inactivation in mutants of Shaker potassium channels by a peptide derived from ShB. Science 250: 568.

Zimmerberg, J., F.S. Cohen, and A. Finkelstein. 1980. Micromolar $\mathrm{Ca}^{2+}$ stimulates fusion of lipid vesicles with planar bilayers containing a calcium-binding protein. Science 210: 906. 